\title{
Proximity to the Treating Centre and Outcomes Following Subarachnoid Hemorrhage
}

\author{
Cian J. O’Kelly, Julian Spears, David Urbach, M. Christopher Wallace
}

\begin{abstract}
Background: In the management of subarachnoid hemorrhage (SAH), the potential for early complications and the centralization of limited resources often challenge the delivery of timely neurosurgical care. We sought to determine the impact of proximity to the accepting neurosurgical centre on outcomes following aneurysmal SAH. Methods: Using administrative data, we analyzed patients undergoing treatment for aneurysmal subarachnoid hemorrhage at neurosurgical centres in Ontario between 1995 and 2004. We compared mortality for patients receiving treatment at a centre in their county (in-county) versus those treated from outside counties (out-of-county). We also examined the impact of distance from the patient's residence to the treating centre. Results: The mortality rates were significantly lower for in-county versus out-of-county patients $(23.5 \%$ vs. $27.6 \%, \mathrm{p}=0.009)$. This advantage remained significant after adjusting for potential confounders $(\mathrm{HR}=0.84, \mathrm{p}=0.01)$. The relationship between distance from the treating centre and mortality was biphasic. Under $300 \mathrm{~km}$, mortality increased with increasing distance. Over $300 \mathrm{~km}$, a survival benefit was observed. Conclusions: Proximity to the treating neurosurgical centre impacts survival after aneurysmal SAH. These results have significant implications for the triage of these critically ill patients.
\end{abstract}

RÉSUMÉ: Proximité du centre de traitement et résultats cliniques suite à une hémorragie sous-arachnoïdienne. Contexte : Dans le traitement de l'hémorragie sous-arachnoïdienne (HSA), les complications précoces et la centralisation de ressources limitées constituent souvent un défi quand il s'agit de procurer des soins neurochirurgicaux en temps opportun. Nous avons évalué l'impact de la proximité du centre de soins neurochirurgicaux sur l'issue clinique après une HSA anévrismale. Méthodes : Nous avons analysé les données administratives de patients qui ont été traités pour une HSA anévrismale dans des centres de neurochirurgie en Ontario entre 1995 et 2004. Nous avons comparé la mortalité des patients qui ont reçu un traitement dans un centre de leur comté de résidence par rapport à ceux qui ont été traités en dehors de leur comté. Nous avons également examiné l'impact de la distance entre la résidence du patient et le centre de traitement. Résultats : Les taux de mortalité étaient significativement plus bas chez les patients qui avaient été traités dans leur comté par rapport à ceux qui avaient été traités en dehors de leur comté $(23,5 \%$ vs $27,6 \% ; \mathrm{p}=0,009)$. Cet avantage est demeuré significatif après ajustement pour les variantes confondantes potentielles ( $R R=0,84 ; p=0,01)$. La relation entre la distance du centre de traitement et la mortalité était biphasique. À moins de 300 km, la mortalité augmentait avec l'augmentation de la distance. Au-delà de 300 km, nous avons observé un bénéfice sur la survie. Conclusions : La proximité du centre de traitement neurochirurgical a un impact sur la survie après une HSA anévrismale. Ces résultats ont des implications significatives pour le triage de ces patients en état critique.

Can. J. Neurol. Sci. 2011; 38: 36-40

Neurosurgical care is generally delivered from a limited number of urban centres. This creates significant challenges for the timely management of urgent neurosurgical cases. In many jurisdictions, the problem is especially exacerbated by vast geographical coverage areas necessitating lengthy transfers. Subarachnoid hemorrhage (SAH) accounts for a significant portion of transfers to tertiary neurosurgical centres. ${ }^{1}$ Subarachnoid hemorrhage patients can experience a multitude of early complications requiring urgent specialized intervention including acute hydrocephalus $\mathrm{s}^{2-4}$, intracerebral hemorrhage ${ }^{5,6}$, cerebral infarction $^{7}$ global edema, cardiopulmonary instability ${ }^{8}$, and aneurysm re-hemorrhage ${ }^{9-12}$. Given their propensity for early deterioration, analysis of the impact of prolonged transfers in this patient group is particularly germane.
The province of Ontario, Canada epitomizes these difficulties in neurosurgical coverage. The province covers a large geographical area, with neurosurgery concentrated within ten urban centres. Using Ontario administrative data, we generated a cohort of patients undergoing neurosurgical intervention for

From the Division of Neurosurgery (CJO), University of Alberta, Edmonton, Alberta; Division of Neurosurgery (JS, MCW), Division of General Surgery (DU), University of Toronto, Toronto, Ontario, Canada.

Received April 27, 2010. Final Revisions Submitted June 29, 2010. Correspondence to: Cian O'Kelly, Division of Neurosurgery, Department of Neurosurgery, University of Alberta, 2D2.02 Mackenzie Health Sciences Centre, 8440-112 Street, Edmonton, Alberta, T6G 2B7, Canada. 
aneurysmal subarachnoid hemorrhage. The cohort was analyzed to determine the impact of a patient's proximity to the treating neurosurgical centre on overall morbidity and mortality.

\section{METHODS}

\section{Data Sources}

The data for this study were derived exclusively from administrative databases maintained at the Institute for Clinical and Evaluative Sciences (Toronto, Ontario, Canada). The Canadian Institute for Health Information Discharge Abstract Database (CIHI DAD) provided information regarding hospital admissions and patient diagnosis. Prior to 2002, this database was coded using the International Classification of Disease version 9 (ICD-9); in subsequent years the version 10 (ICD-10) coding structure was used. Procedure information was derived from the Ontario Health Insurance Plan (OHIP) billing database. This database, employing a unique coding structure, captures health care procedures performed in Ontario. The Registered Persons Database details vital statistics for patients including mortality and residence location (first three digits of postal code and Provincial County).

\section{Study Design}

The ICD 9 and 10 codes for SAH (430 and 160 respectively) were used to select SAH admissions from the CIHI DAD. Patients were included in the study if there was a concurrent OHIP billing for aneurysm repair. The study excluded patients less than 18 years-of-age, aneurysms secondary to another central nervous system pathology (arteriovenous malformations, trauma, presumed mycotic aneurysms (ICD)), aneurysm repairs incorporating a vascular bypass procedure (OHIP), and patients who underwent an aneurysm repair procedure within the preceding two years (OHIP). The study included patients with an index aneurysm repair procedure occurring between January 1, 1995 and December 31, 2004. Follow-up continued through March 31, 2005 ensuring a minimum of three months of followup for all patients.

The patient's proximity to the treating neurological centre was estimated in two ways. The county of residence was used to divide the cohort into patients receiving treatment at a centre in their county of residence (in-county) versus those treated from outside counties (out-of-county). In Canada, the first three digits of the postal code define a specific geographic area. These codes were used to estimate more precisely the patient's geographic location. Google Maps ${ }^{\mathrm{TM}}$ software was used to approximate the distance from the patient's residence to the referring neurosurgical centre by calculating the distance between postal code regions and the treating neurosurgical centres. The primary outcome measure was mortality. Patients were also followed for subsequent SAH admissions indicating re-hemorrhage. Other secondary outcomes included: 30-day mortality (OHIP), overall length-of-stay (CIHI DAD), and discharge to a rehabilitation institution (CIHI DAD), and subsequent diagnostic and therapeutic procedures. A number of covariates were available for multivariate adjustment of the analyses, including: patient age and sex, the Deyo adaptation of the Charlson Co-morbidity Index (CIHI DAD). ${ }^{13}$ The OHIP billing database provided aneurysm treatment modality (surgical versus endovascular), ventilation status of the patient on admission as a surrogate for hemorrhage severity, preoperative or intraoperative evacuation of an intracerebral hemorrhage, acute hydrocephalus as reflected by a preoperative insertion of an external ventricular drain, aneurysm location in the anterior or posterior circulation, and aneurysm size greater than $2.5 \mathrm{~cm}$.

\section{Analysis}

All database manipulation and statistical analyses were conducted using SAS statistical software (version 9.2, SAS Institute, USA). The impact of in-county versus out-of-county patients on mortality was analyzed in univariate and multivariate adjusted Cox proportional hazards model. To account for clustering effects within treatment centres, the standard errors of the estimates were adjusted using the sandwich co-variance estimator of Lin and Wei. ${ }^{14}$ Thirty-day mortality discharge to a rehabilitation institution were analyzed using a similarly adjusted logistic regression modelling. These analyses were repeated to determine the impact on absolute distance from the referring centre on the primary and secondary outcomes.

\section{RESUlts}

Between January 1, 1995 and December 31, 2004, a total of 3260 aneurysmal subarachnoid hemorrhage patients had sufficient data to allow analysis. In-county patients accounted for 1360 patients, with 1900 patients coming to the treating neurosurgical centre from out of county. The mean and median distances from the patient's residence to the treating neurosurgical centre were $93 \mathrm{~km}$ and $39 \mathrm{~km}$ respectively. With an average follow-up duration of 4.25 years (range 0.25-10.25 years), overall mortality for the cohort was $25.9 \%$.

Baseline characteristics for the in-county and out-of-county patients are shown in Table 1. Patients were similar with respect

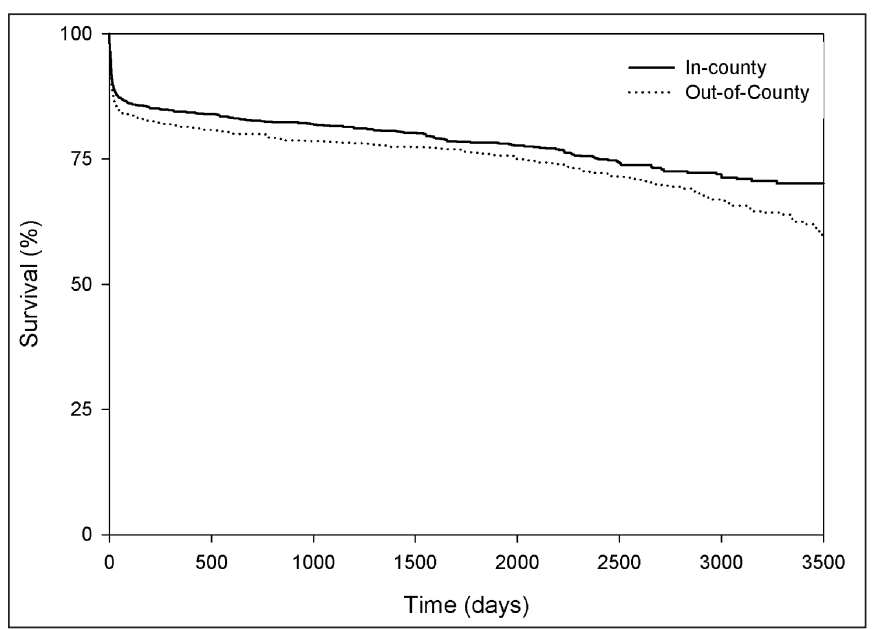

Figure 1: Survival among Ontario residents undergoing repair of a ruptured intracranial aneurysm. Results are stratified according to the location of the patient's residence relative to the treating neurosurgical institution. Patients treated at a neurosurgical centre within their county of residence are designated as 'In-county' (solid line). Patients treated at a neurosurgical centre outside their county of residence are designated as 'Out-of-county' (hatched line). 
Table 1: Mortality and characteristics for in-county and out-of county patients

\begin{tabular}{lcccc}
\hline & In-county & Out-of-county & Total & p-value \\
\hline Numbers & 1360 & 1900 & 3260 & - \\
Mortality & $320(23.5 \%)$ & $524(27.6 \%)$ & $844(25.9 \%)$ & 0.009 \\
Age & 54.2 & 53.9 & 54.1 & 0.56 \\
Charlson index & 0.67 & 0.67 & 0.67 & 0.97 \\
ICH & $15.4 \%$ & $14 \%$ & 14.5 & 0.27 \\
Hydro & $8.4 \%$ & $6.6 \%$ & 7.4 & 0.07 \\
Vent & $8.1 \%$ & $7.2 \%$ & 7.6 & 0.28 \\
Post & $9.7 \%$ & $12.3 \%$ & 11.2 & 0.02 \\
Giant & $3.9 \%$ & $3.2 \%$ & 3.5 & 0.33 \\
Clipping & $77.1 \%$ & $72.5 \%$ & 74.4 & 0.003 \\
\hline
\end{tabular}

ICH - intracerebral hemorrhage on admission, Hydro - acute hydrocephalus, Vent - intubated and ventilated on admission, Posterior - posterior circulation aneurysm repaired, Giant - giant aneurysm repaired, Clipping - index aneurysm repaired by surgical clipping

to age and co-morbidity profile. Subarachnoid hemorrhage severity was not significantly different between groups, as indicated by the proportion of patients ventilated on admission. The out-of-county patients were more likely to have posterior circulation aneurysms. Endovascular coiling was performed more frequently on the out-of-county patients. Kaplan-Meier survival estimates for in-county versus out-of-county patients are shown in Figure 1. Mortality was lower for in-county $(23.5 \%)$ versus out-of-county patients $(27.6 \%)$, corresponding to a hazard ratio (HR) of 0.83 (95\% confidence interval 0.72-0.95, $\mathrm{p}=0.007)$. After adjusting for the baseline characteristics, there remained statistically significant survival benefit for in-county patients (HR=0.84, $\mathrm{p}=0.01)$. Thirty-day mortality was similarly reduced among in-county $(159 / 1360(11.7 \%))$ versus out-of-county (273/1900 (14.4\%)) patients (odds ratio (OR) 0.79 (0.64-0.97), $\mathrm{p}=0.03$ ). Discharge to a rehabilitation hospital was more frequent for in-county $(149 / 1360(11.0 \%))$ versus out-of-county $(102 / 1900(5.4 \%))$ patients (OR 2.16 (1.67-2.82), p<.00001). Hospital length of stay was prolonged in in-county versus out-ofcounty patients ( 30 days versus 22 days, $\mathrm{p}<0.0001$ ).

Absolute distance from the patient's residence to the treating neurosurgical centre did not significantly impact mortality $(\mathrm{HR}=1.00, \mathrm{p}=0.68)$. Analysis of distance intervals indicates a biphasic relationship between distance and mortality. Within $300 \mathrm{~km}$ of the treating neurosurgical centre mortality increases with increasing distance, whereas beyond $300 \mathrm{~km}$ reduced mortality is observed (Figure 2). The baseline characteristics for the distance intervals are shown in Table 2. These parallel the results of the county analysis with overrepresentation of posterior circulation aneurysms, giant aneurysms, and endovascular management in patients transferred from a greater distance.

\section{Discussion}

Our analysis of aneurysmal SAH patients treated in Ontario between 1995 and 2004 indicates that a patient's proximity to the treating neurosurgical centre significantly impacts clinical outcomes. Overall mortality was significantly lower for patients whose residence was in the same county as the treating neurosurgical centre. Sixty-six percent of this excess mortality occurred during the first 30 days, suggesting that early complications may play an important role. Together these results imply that prolonged transfer to the treating neurosurgical centre may contribute to overall mortality for patients with treatable aneurysmal subarachnoid hemorrhage. Furthermore, this effect may be underestimated, given that our analysis was restricted to patient's undergoing aneurysm repair. The study potentially excludes patients who deteriorated in transit and were deemed

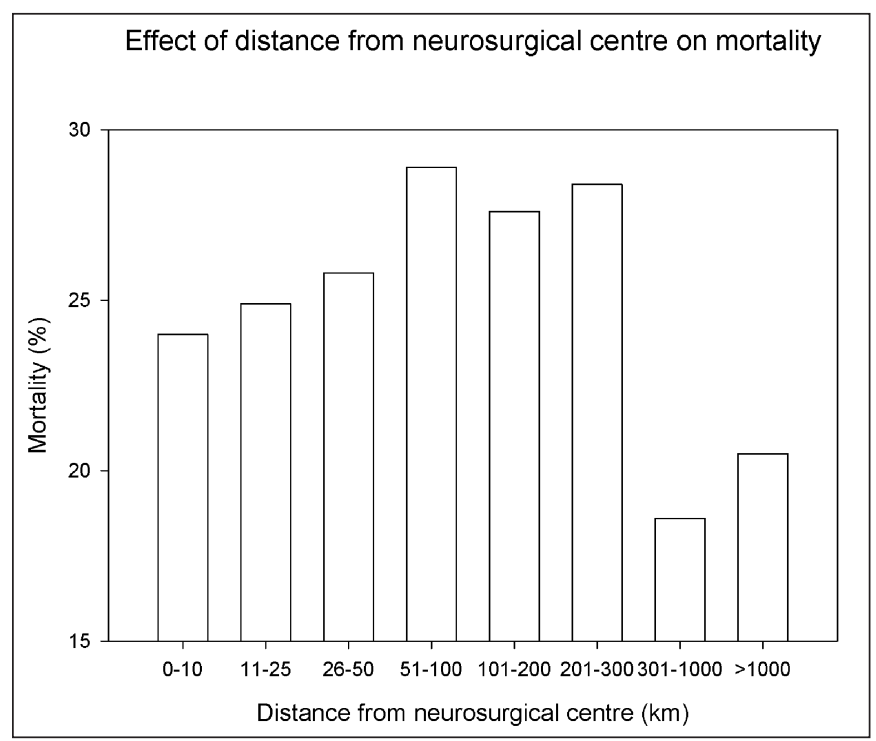

Figure 2: Mortality according to distance from the treating neurosurgical centre. Crude mortality rates are presented for increasing distance intervals from the patient's residence to the treating neurosurgical centre. 
Table 2: Baseline characteristics according to distance from the treating neurosurgical centre

\begin{tabular}{lccccccccc}
\hline Distance & N & $\begin{array}{c}\text { Age } \\
(\mathbf{y r s})\end{array}$ & $\begin{array}{c}\text { Charlson } \\
\text { Index }\end{array}$ & $\begin{array}{c}\text { ICH } \\
(\mathbf{\%})\end{array}$ & $\begin{array}{c}\text { Hydro } \\
(\mathbf{\%})\end{array}$ & $\begin{array}{c}\text { Vent } \\
(\mathbf{\%})\end{array}$ & $\begin{array}{c}\text { Posterior } \\
(\%)\end{array}$ & $\begin{array}{c}\text { Giant } \\
(\mathbf{\%})\end{array}$ & $\begin{array}{c}\text { Clipping } \\
(\mathbf{\%})\end{array}$ \\
\hline $\mathbf{0 - 1 0} \mathbf{~ k m}$ & 630 & 54.1 & 0.6 & 15.7 & 7.1 & 8.7 & 10.6 & 4.1 & 79.7 \\
$\mathbf{1 1 - 2 5} \mathbf{~ k m}$ & 646 & 54.7 & 0.7 & 15.2 & 8.2 & 6.7 & 9.3 & 3.1 & 75.7 \\
$\mathbf{2 6 - 5 0 ~} \mathbf{~ k m}$ & 571 & 53.0 & 0.7 & 15.2 & 9.6 & 5.3 & 10.7 & 3.3 & 74.4 \\
$\mathbf{5 1 - 1 0 0 ~} \mathbf{~ k m}$ & 653 & 55.0 & 0.7 & 13.3 & 7.5 & 5.7 & 11.9 & 4.1 & 72.3 \\
$\mathbf{1 0 1 - 2 0 0 ~} \mathbf{~ k m}$ & 485 & 54.0 & 0.7 & 11.8 & 5.2 & 9.9 & 11.3 & 2.3 & 75.7 \\
$\mathbf{2 0 1 - 3 0 0 ~} \mathbf{~ k m}$ & 102 & 53.8 & 0.8 & 20.6 & 8.8 & 12.8 & 11.8 & 2.9 & 70.6 \\
$\mathbf{3 0 1 - 1 0 0 0 ~} \mathbf{~ k m}$ & 129 & 52.6 & 0.7 & 17.1 & 1.6 & 11.6 & 17.8 & 4.7 & 72.1 \\
$>\mathbf{1 0 0 0} \mathbf{~ k m}$ & 44 & 52.4 & 0.8 & 6.8 & 4.5 & 11.4 & 22.7 & 4.6 & 70.5 \\
\hline
\end{tabular}

ICH - intracerebral hemorrhage on admission, Hydro - acute hydrocephalus, Vent - intubated and ventilated on admission, Posterior - posterior circulation aneurysm repaired, Giant - giant aneurysm repaired, Clipping index aneurysm repaired by surgical clipping

ineligible for aneurysm repair on the basis of poor neurologic grade.

The analysis of absolute distance from the patient's residence to the treating neurosurgical centre underscores the complexity of the overall paradigm. Distance alone did demonstrate a significant linear relationship with mortality, however when specific distance intervals are analyzed increasing mortality is observed for transfers within $300 \mathrm{~km}$ of the referring centre, while transfers longer than $300 \mathrm{~km}$ fared better. This may represent a survival effect where better grade patients, deemed suitable for long distance transfer, are sent for specialized management to high volume centres. This is further reflected by the preponderance of giant aneurysms, posterior circulation locations, and endovascular interventions in this group. Paradoxically, discharge to a rehabilitation institution and length of stay were prolonged in the in-county versus out-of-county transfers. These outcomes are generally used as surrogates for morbidity among surviving patients, however in this instance their surrogacy may be invalid. Out-of-county patients are often transferred to a hospital close to their home to complete rehabilitation whereas in-county patients wait in hospital to attend specialized rehabilitation institutions within the same municipality as the treating neurosurgical centre.

A number of important limitations must be considered in the interpretation of this study. Validity relies on the coding structures within the administrative databases. The OHIP billing database has been shown to accurately capture procedures ${ }^{15}$, while the ICD-9 codes for SAH have excellent measures of validity. ${ }^{16}$ Nevertheless, certain clinical parameters such as grade and neurologic morbidity rely on imperfect surrogate measures, while aneurysm and intervention details are not completely specified. Distance from the referring centre is used as a surrogate for time of transfer. The exact duration of patient transfer was not available from our databases, nor was details of the patients' mode of transfer or level of care at the referring institution. As discussed above, patients with aneurysmal subarachnoid hemorrhage who do not undergo treatment are excluded. These patients are difficult to reliably distinguish from other causes of subarachnoid hemorrhage. Most importantly, we cannot definitively identify the causes of excess mortality among the out-of-county patients. Given these limitations, the results of this study must be considered preliminary and hypothesis generating. We have described a correlation between proximity to the neurosurgical centre and mortality following aneurysmal subarachnoid hemorrhage. In order to demonstrate causality, further research is required.

The transfer of subarachnoid hemorrhage patients is a complex paradigm with multiple independent processes, from the moment a patient enters a referring hospital until they receive definitive intervention at a neurosurgical centre. At the referring hospital, recognition of the diagnosis, time to initial imaging, and medical management can all impact ultimate outcomes. Many studies have detailed the relative utility of various diagnostic tests ${ }^{17}$, however very little has been written with respect to optimizing medical management of these patients while awaiting transfer. Wilson et al propose a simple check list to facilitate non-neurosurgical centre management of $\mathrm{SAH}$ patients, focusing on prevention of early complications ${ }^{18}$. The utility and efficacy of this strategy has not been validated but certainly warrants further attention.

Few studies have directly analyzed SAH transfers. Byrne et al reported on all neurosurgical transfers to three major centres in Cook County, Illinois, with SAH accounting for $30 \%$ of their transfer cohort. ${ }^{1}$ The mean time for transfer was five hours and ten minutes, with a deterioration in Glasgow Coma Score (GCS) noted in $12 \%$ of patients overall. ${ }^{1}$ While numbers for the SAH patients are not reported separately, a five hour delay could result in significant deterioration in this group. Transfer time was not available from our databases, however given that Ontario has a similar population to Illinois and covers ten times the surface area longer transfer times are likely.

Several factors likely contribute to delays and deterioration in neurosurgical patients. Similar to the findings in our study, distance from a trauma centre is correlated with poorer outcomes in closed head injury, especially in remote rural locations. ${ }^{19}$ The availability of specialized transfer mechanisms is likely important, however the relative rapidity of ground versus air, varies depending on specific regions. ${ }^{20}$ Bed availability at 
adjacent neurosurgical centres is a frequent obstacle, compounding transfer delays. ${ }^{1}$ Finally, volume and the degree of endovascular sub-specialization at the accepting neurosurgical centre are recognized as important predictors of outcome in the management of ruptured and unruptured intracranial aneurysms. $^{21-23}$

\section{Conclusions}

A patient's proximity to the treating neurosurgical centre significantly impacts mortality after aneurysmal subarachnoid hemorrhage. The precise cause of this relationship cannot be gleaned from our data. Nevertheless, these findings have important clinical and policy implications for the management, triage, and transfer of these critically ill patients. Further research is required to confirm these results and establish specific factors impacting on outcome, such as time of transfer, mode of transport, and care provided at the referring institution. These factors can then be addressed to optimize transfer systems and minimize the potential impact of inevitable delays.

\section{Funding}

This research was supported by a research fellowship from the Heart and Stroke Foundation of Canada.

\section{REFERENCES}

1. Byrne RW, Bagan BT, Slavin KV, Curry D, Koski TR, Origitano TC. Neurosurgical emergency transfers to academic centers in Cook County: a prospective multicenter study. Neurosurgery. 2008 Mar;62(3):709-16.

2. Kassell NF, Torner JC, Jane JA, Haley EC, Jr., Adams HP. The international cooperative study on the timing of aneurysm surgery. Pt 2: surgical results. J Neurosurg. 1990 Jul;73(1): $37-47$

3. Pietila TA, Heimberger KC, Palleske H, Brock M. Influence of aneurysm location on the development of chronic hydrocephalus following SAH. Acta Neurochir (Wien). 1995;137(1-2):70-3.

4. Vale FL, Bradley EL, Fisher WS, 3rd. The relationship of subarachnoid hemorrhage and the need for postoperative shunting. J Neurosurg. 1997 Mar;86(3):462-6.

5. Nakagawa T, Suga S, Mayanagi K, Akaji K, Inamasu J, Kawase T. Predicting the overall management outcome in patients with a subarachnoid hemorrhage accompanied by a massive intracerebral or full-packed intraventricular hemorrhage: a 15year retrospective study. Surg Neurol. 2005 Apr;63(4):329-34; discussion 334-5.

6. Shimoda M, Oda S, Shibata M, Tominaga J, Kittaka M, Tsugane R. Results of early surgical evacuation of packed intraventricular hemorrhage from aneurysm rupture in patients with poor-grade subarachnoid hemorrhage. J Neurosurg. 1999 Sep;91(3):408-14.
7. Schmidt JM, Rincon F, Fernandez A, et al. Cerebral infarction associated with acute subarachnoid hemorrhage. Neurocrit Care. 2007;7(1):10-7.

8. Wartenberg KE, Mayer SA. Medical complications after subarachnoid hemorrhage: new strategies for prevention and management. Curr Opin Crit Care. 2006 Apr;12(2):78-84.

9. Gallhofer B, Auer LM. Spontaneous course after subarachnoid haemorrhage--evaluation of 109 patients. Acta Neurochir (Wien). 1982;63(1-4):67-70.

10. Nishioka H. Report on the cooperative study of intracranial aneurysms and subarachnoid hemorrhage. section vii. i. evaluation of the conservative management of ruptured intracranial aneurysms. J Neurosurg. 1966 Nov;25(5):574-92.

11. Phillips LH, 2nd, Whisnant JP, O'Fallon WM, Sundt TM, Jr. The unchanging pattern of subarachnoid hemorrhage in a community. Neurology. 1980 Oct;30(10):1034-40.

12. Winn HR, Almaani WS, Berga SL, Jane JA, Richardson AE. The long-term outcome in patients with multiple aneurysms. Incidence of late hemorrhage and implications for treatment of incidental aneurysms. J Neurosurg. 1983 Oct;59(4):642-51.

13. Deyo RA, Taylor VM, Diehr P, et al. Analysis of automated administrative and survey databases to study patterns and outcomes of care. Spine. 1994 Sep 15;19 Suppl 18:2083S-91S.

14. Lin DY, Wei LJ. The robust inference for the proportional hazards model. J Am Stat Assoc. 1989;84:1074-8.

15. Hawker GA, Coyte PC, Wright JG, Paul JE, Bombardier C. Accuracy of administrative data for assessing outcomes after knee replacement surgery. J Clin Epidemiol. 1997 Mar;50(3): 265-73.

16. Tirschwell DL, Longstreth WT, Jr. Validating administrative data in stroke research. Stroke. 2002 Oct;33(10):2465-70.

17. van Gijn J, Rinkel GJ. Subarachnoid haemorrhage: diagnosis, causes and management. Brain. $2001 \mathrm{Feb}$;24(Pt 2):249-78.

18. Wilson SR, Hirsch NP, Appleby I. Management of subarachnoid haemorrhage in a non-neurosurgical centre. Anaesthesia. 2005 May;60(5):470-85.

19. Gonzalez RP, Cummings G, Mulekar M, Rodning CB. Increased mortality in rural vehicular trauma: identifying contributing factors through data linkage. J Trauma. 2006 Aug;61(2):404-9.

20. Karanicolas PJ, Bhatia P, Williamson J, et al. The fastest route between two points is not always a straight line: an analysis of air and land transfer of nonpenetrating trauma patients. J Trauma. 2006 Aug;61(2):396-403.

21. Bardach NS, Zhao S, Gress DR, Lawton MT, Johnston SC. Association between subarachnoid hemorrhage outcomes and number of cases treated at California hospitals. Stroke. 2002 Jul; 33(7): 1851-6.

22. Cross DT, 3rd, Tirschwell DL, Clark MA, et al. Mortality rates after subarachnoid hemorrhage: variations according to hospital case volume in 18 states. J Neurosurg. 2003 Nov;99(5):810-7.

23. Hoh BL, Rabinov JD, Pryor JC, Carter BS, Barker FG, 2nd. Inhospital morbidity and mortality after endovascular treatment of unruptured intracranial aneurysms in the United States, 19962000: effect of hospital and physician volume. AJNR Am J Neuroradiol. 2003 Aug;24(7):1409-20. 